\title{
In vitro Antifungal Effects of Hesperetin and Silibinin
}

\author{
Ramar Gowtham ${ }^{1}$, Fysal Yousuf MA ${ }^{1}$, Devaraj Ezhilarasan ${ }^{*}$, Shanmugam Sambantham², Balakrishnan Anandan²
}

\section{Ramar Gowtham ${ }^{1}$, Fysal Yousuf $\mathrm{MA}^{1}$, Devaraj Ezhilarasan ${ }^{1 *}$, Shanmugam Sambantham², Balakrishnan Anandan²}

'Department of Pharmacology, Saveetha Dental College and Hospital, Saveetha Institute of Medical and Technical Sciences, Saveetha University, Chennai, Tamil Nadu, INDIA-600 077. ${ }^{2}$ Department of Genetics, University of Madras, Chennai, Tamil Nadu, INDIA.

Correspondence

Devaraj Ezhilarasan,

M.Sc., M.Sc., Ph.D.,

Assistant Professor, Department of Pharmacology, Saveetha Dental College and Hospitals, Saveetha Institute of Medical and Technical Sciences, Saveetha University, Chennai,

Tamil Nadu, INDIA -600 077.

Phone No: 9944008201

E-mail: ezhild@gmail.com

\section{History}

- Submission Date: 05-12-2017.

- Review completed: 05-03-2018;

- Accepted Date: 03-05-2018

\section{DOI : 10.5530/pj.2018.4.133}

Article Available online http://www.phcogj.com/v10/i4

\section{Copyright}

(C) 2018 Phcog.Net. This is an openaccess article distributed under the terms of the Creative Commons Attribution 4.0 International license.

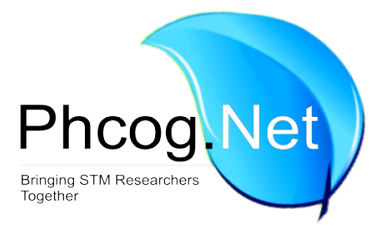

\begin{abstract}
Background: Increasing resistance of microorganisms against available antimicrobial agents is of major concern among scientists and clinicians worldwide. To overcome the resistance and other drawbacks of the current antimicrobial drugs and to obtain more efficacious drugs, an antimicrobial drug having a novel mode of action should be developed. The flavonoid compounds have demonstrated for their therapeutic potential as antimicrobial agents previously. Aim: To evaluate the antifungal efficacy of plant derived compounds such as hesperetin (HSP) and silibinin (SBN) compounds in vitro. Materials and Methods: In this study, we evaluated the antifungal effect of two plant derived flavonoid compounds i.e., SBN and HSP against Candida albicans, Candida tropicalis, Malassezia furfur, Aspergillus niger, Penicillium oxalicum. The disk diffusion test was performed to test the antifungal effect of HSP and SBN with three different concentrations i.e., 15, 20, $25 \mathrm{mg} / \mathrm{ml}$ of HSP and SBN. The antifungal efficacy was studied for $72 \mathrm{~h}$. Results: Our results indicate that SBN has significant antifungal effect than that of HSP. The high sensitivity of these compounds was found against $C$. albicans, $C$. tropicalis. Conclusion: These drugs could be good candidate to combine with regular antimicrobial agents.

Key words: Flavonoids, Silibinin, Hesperetin, Candida albicans, Fungal infection.
\end{abstract}

\section{INTRODUCTION}

A new spectrum of human fungal infections is increasing due to increased cancer, AIDS, and immunocompromised patients. The increased use of antifungal agents also resulted in the development of resistance and it makes necessary to discover new classes of antifungal compounds to cure fungal infections. ${ }^{1}$ Candida albicans is a polymorphic fungus which is the predominant cause of invasive candidiasis, superficial and deep tissue fungal infections. In particular, the invasive candidiasis is the most common invasive fungal disease causing an unacceptably high mortality. Some species of Candida have emerged as an important cause of severe candidaemia and can exhibit reduced susceptibility to the current antifungal agents. ${ }^{2}$ Meanwhile, candidiasis due to Candida tropicalis has increased dramatically on a global scale thus proclaiming this organism to be emerging pathogenic yeast.

Candida tropicalis is one of the more common Candida causing human diseases in tropical countries and it has been identified as the most prevalent pathogenic yeast species of the Candida-non-albicans group. ${ }^{3}$ Resistance to fluconazole in clinical isolates of $C$. tropicalis has increased. ${ }^{4,5}$ There are several reports on azole resistance is reported, specifically in C. albicans and C. tropicalis. ${ }^{6,7}$ The Malassezia comprises lipophilic species, the natural habitat is the skin of humans and other warm-blooded animals. ${ }^{8}$ Malassezia species can be involved in skin disorders, such as pityriasis versicolor, seborrheic dermatitis, atopic eczema, and folliculitis, and occur at higher population densities on scalps with dandruff than on scalps without dandruff.9 Penicillium oxalicum, a plant pathogen and it has been recently found that causes an opportunistic fungal infection in patients with acute myeloid leukemia, diabetes mellitus, and chronic obstructive pulmonary disease. ${ }^{10}$

Increasing resistance of microorganisms against available antimicrobial agents is of major concern among scientists and clinicians worldwide. It is reported that pathogenic viruses, bacteria, fungi, and protozoa are more and more difficult to treat with the existing drugs due to development of resistant. ${ }^{11}$ To overcome the drawbacks of the current antimicrobial drugs and to obtain more efficacious drugs, an antimicrobial drug having a novel mode of action should be developed. Plant-derived flavonoids are a large group of naturally occurring phenylchromones found in fruits, vegetables, tea, and wine. Drugs derived from natural sources play a significant role in the prevention and treatment of human diseases. ${ }^{12}$ Hence, in this study, we tested the antifungal effect of hesperetin and silibinin against different fungal species.

Silibinin (SBN) is a flavonolignan extracted from the seeds of a medicinal plant Silybum marianum (Asteraceae), commonly called "milk thistle"." Hesperetin (HSP) is isolated from citrus fruits that have previously been found to exert anticancer activity through a variety of mechanisms. ${ }^{14}$ Both HSPandSBN(Figure1) havebeenreported tohavewide spectrum of biological activities. These plant derived 
flavonoid compounds have been tested for their anti-proliferative, antimicrobial, anti-inflammatory, antioxidant, anticancer, free radical scavenging, membrane stabilizing properties and came out with promising results. ${ }^{14-17}$ However, studies regarding their antifungal effect are scanty. Hence, in the present study we evaluated the antifungal efficacy of hesperetin and silibinin against different fungal species.

\section{MATERIALS AND METHODS}

\section{Reagents and Drugs}

Hesperetin (C16H14O6; CAS No. 69097-99-0) was purchased from Sigma Chemicals, India.

Silibinin $\left(\mathrm{C}_{25} \mathrm{H}_{22} \mathrm{O}_{10}\right.$; CAS No. 22888-70-6) was purchased from Sigma Chemicals, India. It is a mixture of two diastereomers (silybin A and silybin B). All other chemical used in this study purchased locally and were of analytical grade.

\section{Fungal species used}

Candida albicans, Candida tropicalis, Malassezia furfur, Aspergillus niger, Penicillium oxalicum

\section{Experimental design}

The fungal cultures in Sabouraud's Dextrose Agar plate were filled with three different concentrations i.e., 15, 20, $25 \mathrm{mg} / \mathrm{ml}$ of HSP and SBN. The antifungal efficacy was studied for $72 \mathrm{~h}$.

\section{Disk diffusion method}

The disk diffusion test was performed to test the antifungal effect of HSP and $\mathrm{SBN}^{18}$ On sterile plates containing Sabouraud's Dextrose Agar, the fungal cultures were swabbed. Then, sterile discs (made from Whatman filter paper) each about $5 \mathrm{~mm}$ diameter impregnated with the HSP and SBN separately was placed on the inoculated plates. Similarly, each plate was placed with a sterile disc. The plates were incubated at $28^{\circ} \mathrm{C}$ for $72 \mathrm{~h}$ for evaluation. The diameter of inhibition zones formed around the disk was measured in millimeter. The test was carried out in triplicate in each run of the experiments.

\section{RESULTS}

\section{Antifungal activity of HSP}

The results from this study clearly show that HSP has antifungal efficacy only against C. albicans, C. tropicalis, Malassezia furfur species. Hesperetin has produced dose-dependent inhibition of C. tropicalis and Malassezia furfur fungal growth, whereas, in low concentrations hesperetin did not show any effect in the growth of $C$. albicans. The maximum inhibitory effect was seen at maximum dose tested in this study i.e., $25 \mathrm{mg} / \mathrm{ml}$. This flavonoid exhibits higher efficacy against C. tropicalis and Malassezia furfur when compared to C. albicans (Figure 2). Hesperetin did show any significant change in the growth of Aspergillum niger and Penicillium oxalicum.

\section{Antifungal activity of SBN}

We also tested the antifungal efficacy of the SBN against the C. albicans, C. tropicalis, Malassezia furfur, Aspergillum niger and Penicillium oxalicum. The results obtained from present study shows that SBN has antifungal efficacy only against C. albicans, C. tropicalis, Malassezia furfur among fungal organism tested. Silibinin shows high sensitivity against Candida species i.e., C. albicans, C. tropicalis when compared to other species (Figure 3). In fact, SBN did show any significant change in the growth of Aspergillum niger and Penicillium oxalicum. The maximum effect was observed with higher concentration of SBN used in this study.

\section{DISCUSSION}

Human fungal pathogens cause invasive infections are hidden killers, taking lives of 1.5 million people every year. ${ }^{19}$ Therapeutic applications of immunosuppressive drugs, the use of broad spectrum antibiotics against various clinical settings are responsible for an increasing number of immunocompromised patients and consequent opportunistic fungal infections worldwide. An impaired immune system provides favorable conditions for pathogenic and non-pathogenic micro-organisms. ${ }^{3}$

Hesperetin, a flavanone glycoside predominantly found in citrus fruits, exhibits a wide array of biological properties. ${ }^{20}$ Studies have shown the antimicrobial effect of HSP especially against bacterial strains and came out with promising results. ${ }^{21}$ In the present study, HSP shows antifungal activity against Candida species i.e., C. albicans (only in higher concentration) and C. tropicalis. The HSP treatment also shows inhibitory effect against Malassezia furfur. On the other hand antimicrobial effect of SBN has also been concretely proved against wide variety of bacterial
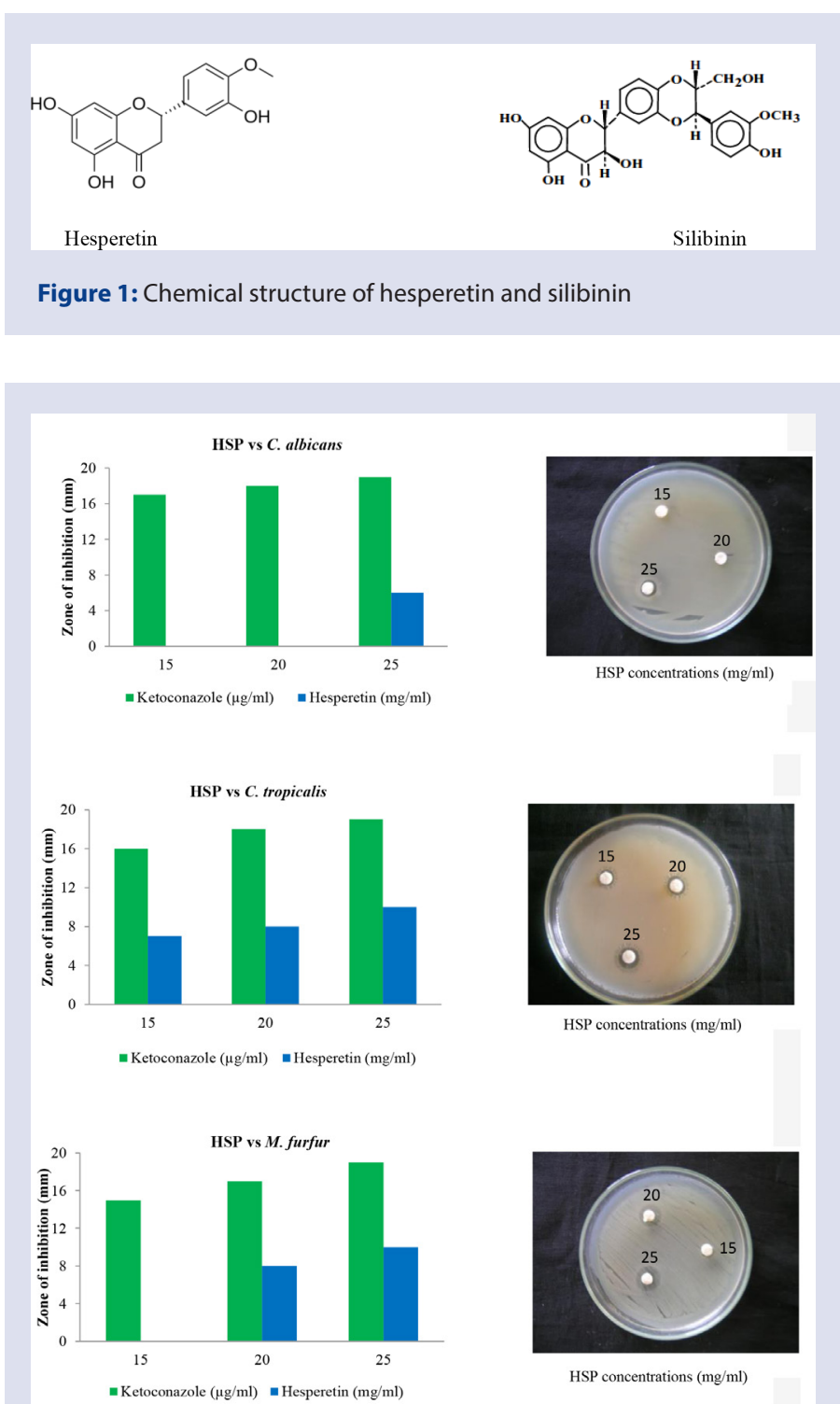

HSP concentrations $(\mathrm{mg} / \mathrm{ml})$

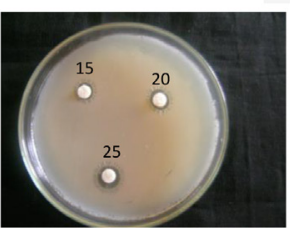

HSP concentrations (mg/ml)

Figure 2: Antifungal effect of hesperetin. 


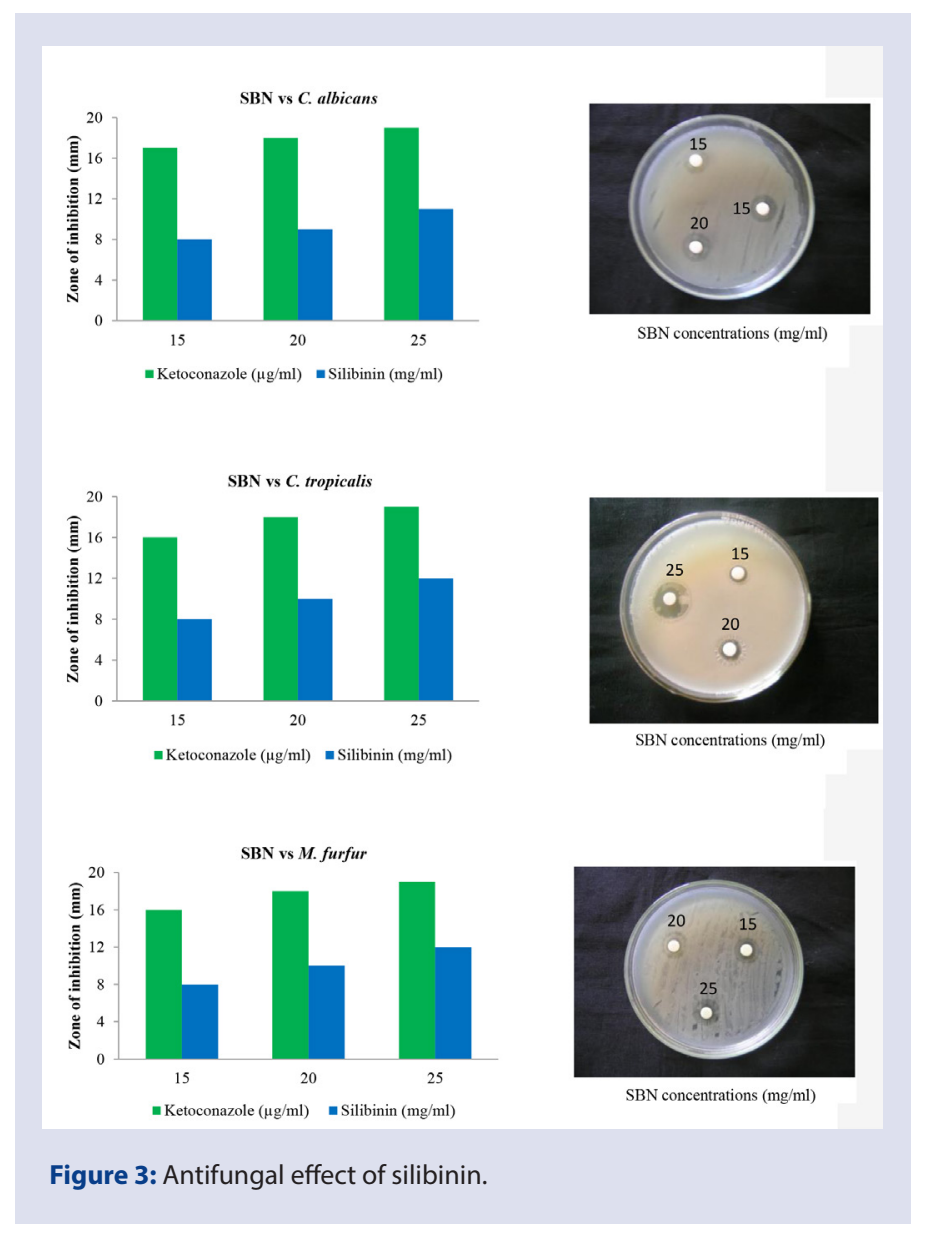

strains. ${ }^{17,22}$ The antifungal effect of SBN found in this study is marginally greater than HSP. Moreover, SBN shows higher efficacy against C. albicans and C. tropicalis.

Flavonoids are hydroxylated phenolic substances synthesized by plants in response to microbial infection. Flavones are phenolic structures containing one carbonyl group and the addition of a 3-hydroxyl group yields a flavonol. Flavonoids have been found to be effective antimicrobial substances against a wide array of microorganisms. Their activity is probably was suggested due to their ability to complex with extracellular and soluble proteins and to complex with fungal cell wall. It also been said that high lipophilic nature of flavonoids also disrupts fungal membranes. ${ }^{23}$ In light of the above reports it is suggested that the profound antifungal effect of HSP and SBN found in this study could be due to their action on fungal membrane disruption and subsequent inhibition of its growth.

Collectively, this study clearly shows that SBN possess significant antifungal efficacy than HSP. It should be brought to light that both these flavonoids show efficacy only against C. albicans, C. tropicalis and Malassezia furfur and did not show any efficacy against the Aspergillum niger and Penicillium oxalicum. Furter, it has to emphasize that in previous studies flavonoid compounds have demonstrated for their therapeutic potential as antimicrobial agents. The synergistic effect of flavonoids combined with commonly utilized antibiotics is well supported in the literature and this strategy is emerging as an important complementary treatment modality in research. ${ }^{17}$ Accordingly, the present study would be of immensely useful to identify some of the efficacious plant derived flavonoid compounds which possess antifungal effect.

\section{CONCLUSION}

In conclusion, this study clearly indicates the fact that SBN has the significant antifungal effect against Candida species when compared to HSP. These drugs could be good candidate to combine with regular antimicrobial agents. However, their exact role behind the antifungal effect is further warranted.

\section{CONFLICT OF INTEREST}

The authors declare no conflict of interest.

\section{ABBREVIATIONS}

SBN: Silibinin; HSP: Hesperetin; CAS: Chemical Abstracts Service; AIDS: Acquired Immune Deficiency Syndrome.

\section{REFERENCES}

1. Arif T, Bhosale JD, Kumar N, Mandal TK, Bendre RS, Lavekar GS, et al. Natural products-antifungal agents derived from plants. J Asian Nat Prod Res. 2009;11(7):621-8.

2. Pemán J, Quindós G. Current aspects of invasive diseases caused by Candida and other yeast fungi. Rev Iberoam Micol. 2016;33(3):133-9.

3. Kothavade RJ, Kura MM, Valand AG, Panthaki MH. Candida tropicalis: Its prevalence, pathogenicity and increasing resistance to fluconazole. J Med Microbiol 2010;59(8):873-80

4. Myoken Y, Kyo T, Fujihara M, Sugata T, Mikami Y. Clinical significance of breakthrough fungemia caused by azole-resistant Candida tropicalis in patients with hematologic malignancies. Haematologica. 2004;89(3):378-80.

5. Tortorano AM, Rigoni AL, Biraghi E, Prigitano A, Viviani MA. FIMUA-ECMM Candidaemia Study Group.The European Confederation of Medical Mycology (ECMM) survey of candidaemia in Italy: antifungal susceptibility patterns of 261 non-albicans Candida isolates from blood. J Antimicrob Chemother. 2003;52(4):679-82.

6. Brun S, Bergès T, Poupard P, Vauzelle-Moreau C, Renier G, Chabasse D, et al. Mechanisms of azole resistance in petite mutants of Candida glabrata. Antimicrob Agents Chemother. 2004;48(5):1788-96.

7. Vermitsky JP, Edlind TD. Azole resistance in Candida glabrata: coordinate upregulation of multidrug transporters and evidence for a Pdr1-like transcription factor. Antimicrob Agents Chemother. 2004;48(10):3773-81.

8. Prohic A, Jovovic Sadikovic T, Krupalija-Fazlic M, Kuskunovic-Vlahovljak S. Malassezia species in healthy skin and in dermatological conditions. Int $J$ Dermatol. 2016;55(5):494-504

9. Gemmer CM, De Angelis YM, Theelen B, Boekhout T, Dawson Jr TL. Fast, noninvasive method for molecular detection and differentiation of Malassezia yeast species on human skin and application of the method to dandruff microbiology. J Clin Microbiol. 2002;40(9):3350-7.

10. Chowdhary A, Kathuria S, Agarwal K, Sachdeva N, Singh PK, Jain S, et al. Voriconazole-Resistant Penicillium oxalicum: An Emerging Pathogen in Immunocompromised Hosts. Open Forum Infect Dis. 2014;1(2):ofu029.

11. Koomen GJ, Den Blaauwen T, Hellingwerf KJ, Ungaro R, Mobashery S. Fighting microbial resistance through development of new antimicrobial agents, directed against new specific targets. IUPAC Project. 2002:030-1.

12. Ezhilarasan D, Sokal E, Karthikeyan S, Najimi M. Plant derived antioxidants and antifibrotic drugs: Past, Present and Future. J Coast Life Med. 2014;2:738-45.

13. Ezhilarasan D, Evraerts J, Sid B, Calderon PB, Karthikeyan S, Sokal E, et al. Silibinin induces hepatic stellate cell cycle arrest via enhancing p53/p27 and inhibiting Akt downstream signaling protein expression. Hepatobiliary Pancreat Dis Int. 2017;16(1):80-87.

14. Sambantham S, Radha M, Paramasivam A, Anandan B, Malathi R, Chandra $\mathrm{SR}$, et al. Molecular mechanism underlying hesperetin-induced apoptosis by in silico analysis and in prostate cancer PC-3 cells. Asian Pac J Cancer Prev. 2013;14(7):4347-52.

15. Ezhilarasan D, Karthikeyan S. Silibinin alleviates $N$-nitrosodimethylamineinduced glutathione dysregulation and hepatotoxicity in rats. Chin J Nat Med. 2016;14(1):40-7.

16. Ezhilarasan D, Evraerts J, Brice S, Calderon PB, Karthikeyan S, Sokal E, et al. Silibinin Inhibits Proliferation and Migration of Human Hepatic Stellate LX-2 Cells. 2016. J Clin Exp Hepatol. 2016;6(3):167-74

17. De Oliveira DR, Tintino SR, Braga MF, Boligon AA, Athayde ML, Coutinho HD, et al. In vitro antimicrobial and modulatory activity of the natural products silymarin and silibinin. Biomed Res Int. 2015;2015:292797.

18. Bhalodia NR, Shukla VJ. Antibacterial and antifungal activities from leaf extracts of Cassia fistula I.: An ethnomedicinal plant. J Adv Pharm Technol Res. $2011 ; 2(2): 104-9$ 
19. Kim JY. Human fungal pathogens: Why should we learn? J Microbiol. 2016;54(3):145-8.

20. Palit S, Kar S, Sharma G, Das PK. Hesperetin Induces Apoptosis in Breast Carcinoma by Triggering Accumulation of ROS and Activation of ASK1/JNK Pathway. J Cell Physiol. 2015;230(8):1729-39.

21. Moon SH, Lee JH, Kim KT, Park YS, Nah SY, Ahn DU, et al. Antimicrobial effect of 7-O-butylnaringenin, a novel flavonoid, and various natural flavonoids against
Helicobacter pylori strains. Int J Environ Res Public Health. 2013;10(11):5459-69.

22. Lee YS, Jang KA, Cha JD. Synergistic antibacterial effect between silibinin and antibiotics in oral bacteria. J Biomed Biotechnol. 2012;2012:618081.

23. Tsuchiya H, Sato M, Miyazaki T, Fujiwara S, Tanigaki S, Ohyama M, et al. Comparative study on the antibacterial activity of phytochemical flavanones against methicillin-resistant Staphylococcus aureus. J Ethnopharmacol. 1996:50(1):27-34

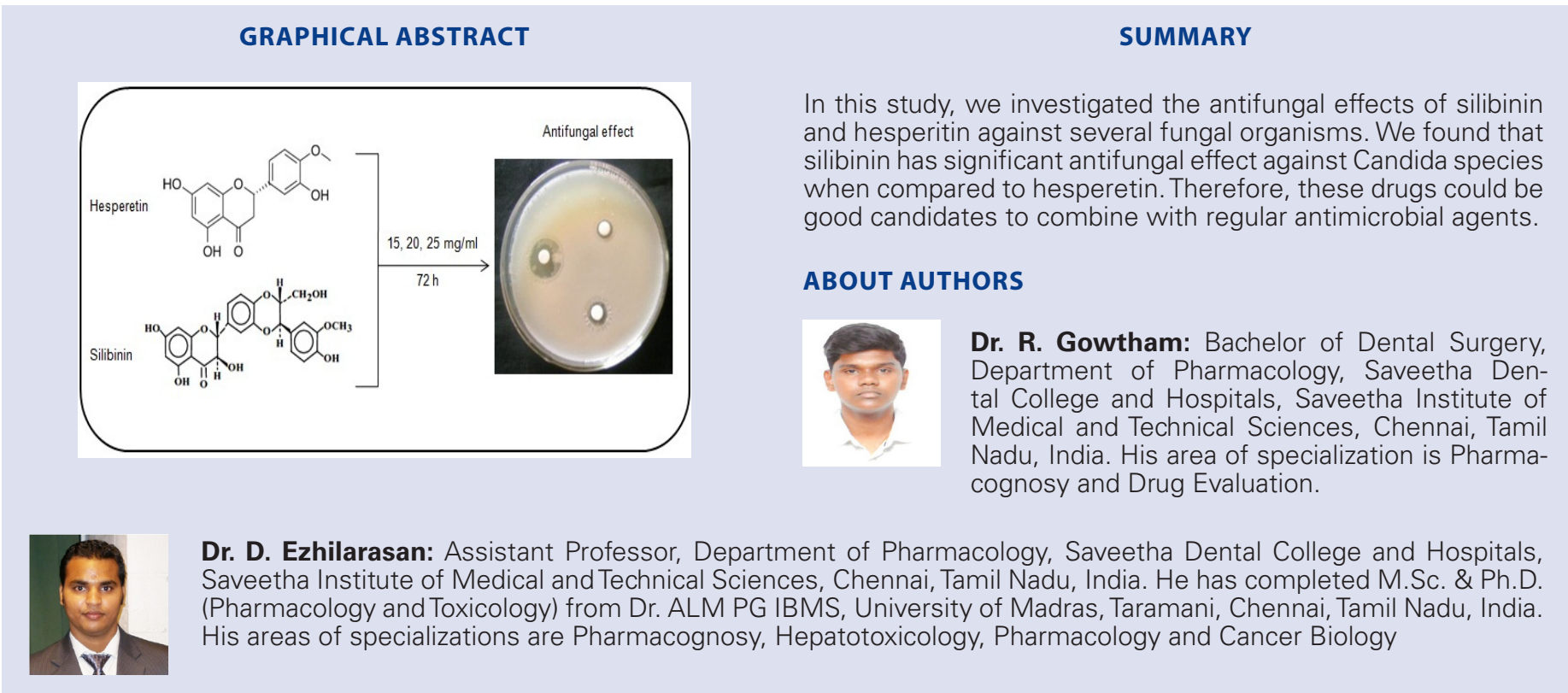

Cite this article: Gowtham R, Yousuf FMA, Ezhilarasan D, Sambantham S, Anandan B. In vitro Antifungal Effects of Hesperetin and Silibinin. Pharmacog J. 2018;10(4):789-92. 